\title{
Suppression of autophagic genes sensitizes CUG2-overexpressing A549 human lung cancer cells to oncolytic vesicular stomatitis virus-induced apoptosis
}

\author{
WARAPORN MALILAS ${ }^{1}$, SANG SEOK KOH ${ }^{2}$, SOOJIN LEE ${ }^{3}$, RATAKORN SRISUTTEE ${ }^{1}$, IL-RAE CHO ${ }^{1}$, \\ JEONG MOON $^{1}$, SIRICHAT KAOWINN ${ }^{1}$, RANDAL N. JOHNSTON ${ }^{4}$ and YOUNG-HWA CHUNG ${ }^{1}$ \\ ${ }^{1}$ BK21+, Department of Cogno-Mechatronics Engineering, Pusan National University, Busan 609-735; \\ ${ }^{2}$ Immunotherapy Research Center, Korea Research Institute of Bioscience and Biotechnology, \\ and Department of Functional Genomics, University of Science and Technology, Daejeon 305-333; \\ ${ }^{3}$ Department of Microbiology and Molecular Biology, Chungnam National University, \\ Daejeon 305-764, Republic of Korea; ${ }^{4}$ Department of Biochemistry and Molecular Biology, \\ University of Calgary, Calgary, T2N4N1 Alberta, Canada
}

Received November 15, 2013; Accepted December 20, 2013

DOI: $10.3892 /$ ijo.2014.2264

\begin{abstract}
We showed in our previous study that cancer upregulated gene $(C U G)$ 2, a novel oncogene, confers resistance to infection of oncolytic vesicular stomatitis virus (VSV) by activating Stat1-mediated signal transduction. Since many studies have reported that autophagy is involved in virus replication, we investigated whether autophagy also plays a role in the antiviral activity in A549 cells overexpressing CUG2 (A549-CUG2). We suppressed Atg5 or Beclin 1 expression using siRNA and examined its effect on the susceptibility of cells to infection by oncolytic VSV. We found that A549-CUG2 cells treated with Atg5 or Beclin 1 siRNA became susceptible to VSV infection, whereas A549-CUG2 cells treated with control siRNA were resistant. This result suggests that autophagy is involved in the antiviral response of A549-CUG2 cells. Further investigation revealed that autophagy impairment enhanced the generation of reactive oxygen species (ROS), which resulted in inactivation of S6 kinase. Under these conditions, the levels of ISG15 transcript and protein decreased, which conferred on A549-CUG2 cell susceptibility to VSV infection. Finally, we found that overloading of $\mathrm{H}_{2} \mathrm{O}_{2}$ sensitized control A549-CUG2 cells to VSV-induced apoptosis. Taken together, these results indicate that autophagy impairment induces excessive ROS formation, which decreases S6 kinase activity and ISG15 expression, ultimately rendering the A549-CUG2 cells susceptible to VSV infection. We propose that autophagy impairment
\end{abstract}

Correspondence to: Professor Young-Hwa Chung, BK21+, Department of Cogno-Mechatronics Engineering, Pusan National University, Busan 609-735, Republic of Korea

E-mail: younghc@pusan.ac.kr

Key words: vesicular stomatitis virus, CUG2, Atg5, Beclin 1, autophagy is a potential strategy for successful VSV virotherapy of CUG2-overexpressing tumors.

\section{Introduction}

Cancer upregulated gene 2 (CUG2) was identified as a candidate gene that is commonly upregulated in various tumor tissues, such as ovarian, liver, colon, and lung tissues, and is known to play a crucial role in tumorigenesis. CUG2 was mapped to chromosome $6 \mathrm{q} 22.32$; it spans $\sim 8.5 \mathrm{~kb}$ with a three-exon structure and encodes an 88 -amino-acid polypeptide (1). Further study revealed that CUG2 is a new centromere component that is required for proper kinetochore function during cell division (2). CUG2 has been shown to exert an oncogenic effect in a transplanted model using NIH3T3 cells expressing CUG2, in a manner similar to Ras (1). Whereas CUG2 overexpression has been shown to activate Ras and MAPKs including p38 MAPK, which eventually facilitates oncolytic reoviral replication (3), our previous studies showed that CUG2 confers resistance to oncolytic vesicular stomatitis virus (VSV) infection (4), as well as induces cell migration and drug resistance (4), through activation of Stat1 (5).

Autophagy is an ancient catabolic process necessary in maintaining homeostasis in eukaryotic cells, whereby longlived cytoplasmic proteins and organelles are degraded and nutrients are provided under starvation or stress conditions (6). It is a programmed, sequential process mainly involving autophage-related gene (Atg) products and has roles in biological processes including development, aging, and degeneration (7). Aberrant regulation of autophagy is associated with many diseases, such as cancer and neurodegenerative diseases $(8,9)$. Initial reports associating autophagy with cancer showed that allelic loss of the essential autophagy gene Beclin 1 (BECNI) is prevalent in human breast, ovarian, and prostate cancers (10) and that BecnI ${ }^{+/-}$mice develop mammary gland hyperplasias, lymphomas, and lung and liver tumors (11). Subsequent 
studies demonstrated that $\operatorname{Atg} 5^{-/-}$and $\operatorname{Atg} 7^{-/-}$mice develop liver adenomas (12). In addition, autophagy has been reported to be involved in virus replication (13-15). Autophagy induction requires the antiviral eIF2 $\alpha$ kinase signaling pathway (including PKR and eIF $2 \alpha$ ), and eIF2 $\alpha$ kinase signaling is antagonized by the herpes simplex virus (HSV-1) neurovirulence gene product ICP34.5 (16). Autophagy plays a key role in the recognition of certain RNA viruses by delivering viral replication intermediates through Toll-like receptor-7 (TLR-7) activation in plasmacytoid dendritic cells (pDCs) (17) and other cells via retinoic acid-inducible gene I (RIG-I) and melanoma differentiation-associated gene 5 (MDA-5) (18-20).

This study aimed to determine whether autophagy is also involved in resistance to oncolytic VSV in A549-CUG2 cells. We show that autophagy impairment induced reactive oxygen species (ROS) formation, which led to decreased ISG15 production due to overexpression of CUG2, eventually sensitizing the cells to VSV-induced apoptosis. We propose that autophagy impairment is a potential strategy for successful VSV virotherapy of CUG2-overexpressing tumors.

\section{Materials and methods}

Cell cultures and virus amplification. Human lung cancer A549 cells stably transfected with CUG2 expression plasmid (A549-CUG2) were used. The cells were cultured in RPMI-1640 supplemented with $10 \%$ fetal bovine serum (FBS), $1 \%$ penicillin, $1 \%$ streptomycin, and puromycin $(0.5 \mu \mathrm{g} / \mathrm{ml})$ at $5 \% \mathrm{CO}_{2}$ and $37^{\circ} \mathrm{C}$. VSV (Indiana strain), purchased from the ATCC (Manassas, VA, USA), was propagated in L929 cells, and viral titer was measured in plaque-forming units.

Reagents and antibodies. For immunoblotting, antibodies against Atg5, ISG15, and poly-ADP-ribose polymerase (PARP) were acquired from Cell Signaling Biotechnology (Danvers, MA, USA). Beclin 1 and $\beta$-actin antibodies were obtained from Santa Cruz Biotechnology (Santa Cruz, CA, USA). VSV glycoprotein (G) antibody was obtained from Abcam (Cambridge, MA, USA).

Western blotting assays. Cells were harvested and lysed in lysis buffer [150 mM NaCl, 1\% NP-40, $50 \mathrm{mM}$ Tris- $\mathrm{HCl}$ (pH 7.5)] containing $0.1 \mathrm{mM} \mathrm{Na} \mathrm{VO}_{3}, 1 \mathrm{mM} \mathrm{NaF}$ and protease inhibitors (Sigma, St. Louis, MO, USA). For immunoblotting, proteins from whole-cell lysates were resolved by 10 or $15 \%$ sodium dodecyl sulfate-polyacrylamide gel electrophoresis (SDS-PAGE) and then transferred onto nitrocellulose membranes. Primary antibodies were used at $1: 1,000$ or $1: 2,000$ dilution, and secondary antibodies conjugated to horseradish peroxidase were used at 1:2,000 dilution in $5 \%$ non-fat dry milk. After the final washing, the nitrocellulose membranes were analyzed by enhanced chemiluminescence assay using the LAS 4000 mini system (Fuji, Tokyo, Japan).

Reverse transcription-polymerase chain reaction. Total RNA was extracted from cells using the RNeasy mini kit (Qiagen, Valencia, CA, USA) according to the manufacturer's instructions. Three micrograms of total RNA was converted to cDNA using Superscript II reverse transcriptase (Invitrogen,
Carlsbad, CA, USA), and polymerase chain reaction (PCR) was performed using the following specific primers: human ISG15, 5'-ATG GGC TGG GAC CTG ACG GTG AAG AT-3' (sense) and 5'-TTA GCT CCG CCC GCC AGG CTC TGT-3'; human IFIT3, 5'-ATG AGT GAG GTC ACC AAG AAT-3' (sense) and 5'-TCA GTT CAG TTG CTC TGA GTT A-3' (antisense). The cDNA for each reaction was diluted, and PCR was run at the optimized cycle number. $\beta$-actin mRNA was also analyzed as an internal standard. After amplification, the products were subjected to electrophoresis on $2.0 \%$ agarose and detected by ethidium bromide staining.

Transfection with short interference RNA. Cells were trypsinized and incubated overnight to achieve $60-70 \%$ confluence before short interference RNA (siRNA) transfection. Atg5 siRNA [500 nM; Bioneer, Daejeon, Korea; sense, 5'-ACU UUG CUG UAA CCC UGU A(dTdT)-3'; antisense, 5'-UAC AGG GUU ACA GCA AAG U(dTdT)-3'], Beclin 1 siRNA [500 nM; Bioneer; sense, 5'-ACU UUG CUG UAA CCC UGU A(dTdT)-3'; antisense, 5'-UAC AGG GUU ACA GCA AAG U(dTdT)-3'], ISG15 siRNA [500 nM; Bioneer; sense, 5'-ACU UUG CUG UAA CCC UGU A(dTdT)-3'; antisense, 5'-UAC AGG GUU ACA GCA AAG U(dTdT)-3'], or negative control siRNA (Bioneer) was mixed with Lipofectamine 2000 (Invitrogen). The cells were incubated with the transfection mixture for $6 \mathrm{~h}$ and then rinsed with RPMI-1640 containing $10 \%$ FBS. The cells were incubated for $48 \mathrm{~h}$ before harvest.

Measurement of ROS. The intracellular ROS levels were determined by using an oxidative-sensitive fluorescence dye, 2',7'-dichlorodihydrofluorescein diacetate (DCF-DA; Molecular Probes, Eugene, OR, USA). Cells were treated with $20 \mu \mathrm{M}$ DCF-DA for $30 \mathrm{~min}$ and then observed under a fluorescence microscope (Carl Zeiss, Axio-D1, Oberkochen, Germany).

\section{Results}

Suppression of Atg5 or Beclin 1 expression sensitizes A549-CUG2 cells to VSV-induced apoptosis. Our previous study showed that CUG2 overexpression induces resistance to VSV infection by activation of the Stat1-2'-5' oligoadenylate synthetase-like 2 (OASL 2) signaling pathway (5). Several lines of evidence have shown that autophagy is implicated with viral replication (13-15), and moreover other studies showed that Atg5-deficient mouse embryonic fibroblast (MEF) cells exhibit resistance to VSV through hyperproduction of type I IFN $(20,21)$. Based on these studies, we tested what role Atg5 protein plays in VSV infection in our cell model. We first optimized the concentration of Atg5 siRNA (500 $\mathrm{nM})$ to significantly reduce Atg5 protein levels in A549-CUG2 cells (Fig. 1A). VSV infection alone did not induce cytolysis in A549-CUG2 cells, as seen in mouse colon cancer cells overexpressing human CUG2 (Fig. 1B). In contrast to previous reports $(20,21)$, A549-CUG2 cells treated with VSV and Atg5 siRNA clearly exhibited cytolysis at $60 \mathrm{~h}$ after infection (Fig. 1B). Control A549 cells stably expressing an empty vector showed acceleration of cytolysis after treatment with VSV and Atg5 siRNA (data not shown). A549-CUG2 cells cotreated with VSV and Atg5 siRNA 


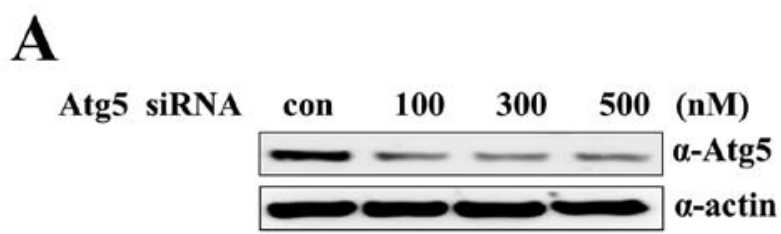

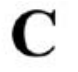

Con
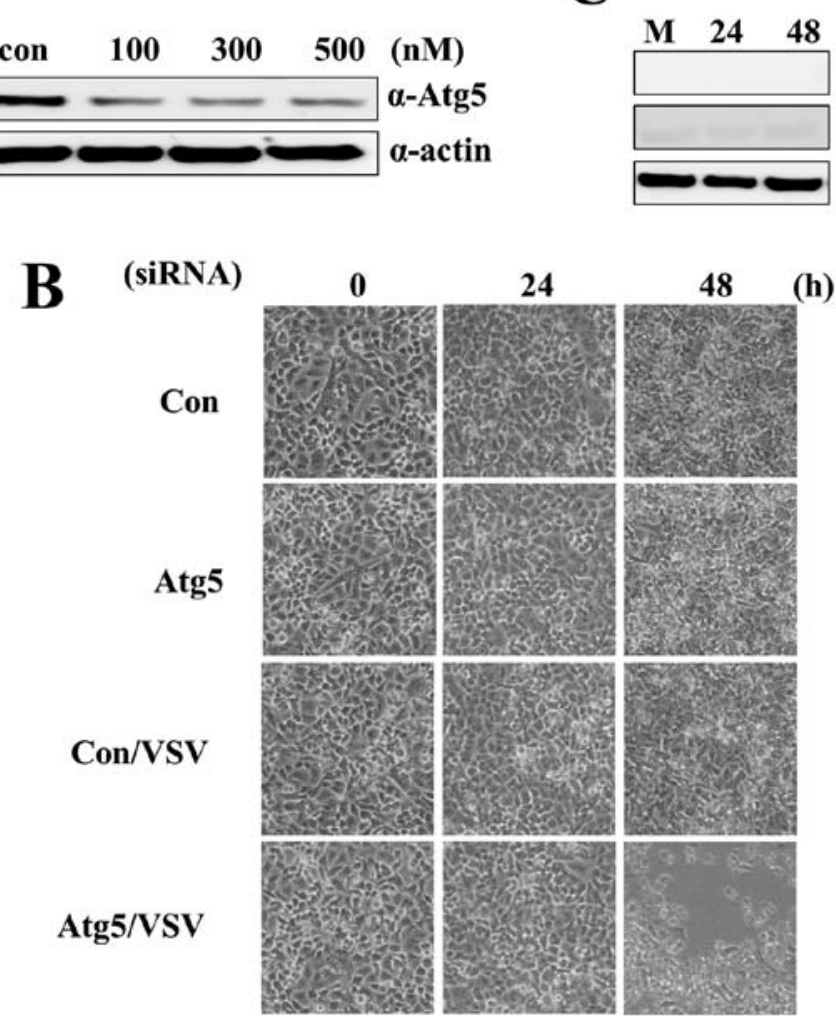

(h)
Atg5 (siRNA/VSV)

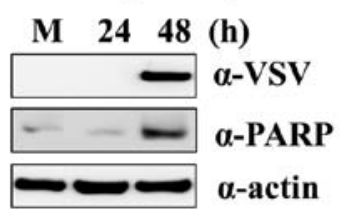

Figure 1. Suppression of Atg5 sensitizes A549-CUG2 cells to VSV-induced apoptosis. (A) A549-CUG2 cells were treated with control or Atg5 siRNA at different concentrations for $48 \mathrm{~h}$, and the expression of Atg5 was examined by immunoblotting with Atg5 antibody. (B) A549-CUG2 cells were treated with control or Atg5 siRNA at $24 \mathrm{~h}$ before VSV infection [multiplicity of infection (MOI) 0.1 ] and observed at 0,24 , and $48 \mathrm{~h}$ after infection under a light microscope. (C) Cell lysates were prepared and separated on a 10\% sodium dodecyl sulfate-polyacrylamide gel electrophoresis (SDS-PAGE) gel. The expression of poly-ADP-ribose polymerase (PARP) and VSV G protein was observed by immunoblotting with the corresponding antibodies.

underwent apoptosis caused by VSV replication (Fig. 1C). These results suggest that autophagy protects A549-CUG2 cells from VSV-induced apoptosis.

To confirm our observation that suppression of autophagic gene expression sensitizes A549-CUG2 cells to VSV-induced apoptosis, we suppressed another autophagic gene, Beclin 1, also known as Atg6. Treatment with Beclin 1 siRNA (500 nM) clearly suppressed Beclin 1 protein expression in A549-CUG2 cells at $48 \mathrm{~h}$ after treatment (Fig. 2A). Cotreatment with VSV and Beclin 1 siRNA induced apoptosis in A549-CUG2 cells (Fig. 2B); the apoptotic cell death was attributed to VSV replication (Fig. 2C). This result also confirmed our hypothesis that autophagy protects A549-CUG2 cells from VSV-induced apoptosis.

Suppression of Atg5 or Beclin 1 expression induces ROS formation in A549-CUG2 cells. Next, we investigated the mechanism by which suppression of Atg5 or Beclin 1 expression sensitizes cells to VSV-induced apoptosis. Recent studies have reported that impaired autophagy in pathogenic diseases including cancer shows accumulated damaged mitochondria and deregulated ROS levels (22). We therefore asked whether suppression of Atg5 or Beclin 1 expression is related to ROS formation in A549-CUG2 cells. To answer this question, we measured, using DCF-DA fluorescence, the ROS levels in A549 cells treated with Atg5 or Beclin 1 siRNA. We found that the cells treated with Atg5 or Beclin 1 siRNA showed enhanced fluorescence compared with control siRNA-treated cells at $48 \mathrm{~h}$ after transfection (Fig. 3A). As a control, A549-CUG2 cells were treated with $\mathrm{H}_{2} \mathrm{O}_{2}$ at different concentrations. We observed that $\mathrm{H}_{2} \mathrm{O}_{2}$ at $20-50 \mu \mathrm{M}$ induced fluorescence intensity similar to that in cells treated with Atg5 or Beclin 1 siRNA (Fig. 3B). These results indicate that the accumulation of damaged mitochondria induced ROS formation in autophagy-deficient cells. Because it was previously reported that the kinase mammalian target of rapamycin (mTOR) stimulates type I IFN production via phosphorylation of its effector proteins including S6 kinase (23), we also examined the activation status of S6 kinase during autophagic suppression in the presence or absence of serum. We found that Atg5 or Beclin 1 suppression decreased the levels of phosphorylated S6 kinase in the presence of serum and more drastic reduction of phosphorylated S6 kinase levels under serumfree conditions (Fig. 3C). The addition of $\mathrm{H}_{2} \mathrm{O}_{2}$ into the medium during culture of A549-CUG2 cells led to an oxidative stress-mediated reduction of S6 kinase phosphorylation, similar to the pattern seen in Atg5 or Beclin 1 suppression (Fig. 3D). This result indicates that oxidative stress caused by autophagy impairment inhibits the activation of S6 kinase, which is an essential step in type I IFN production.

Suppression of ISG15 caused by treatment with Atg5 or Beclin 1 siRNA in A549-CUG2 cells confers susceptibility to VSV infection. Next, we analyzed the association between 


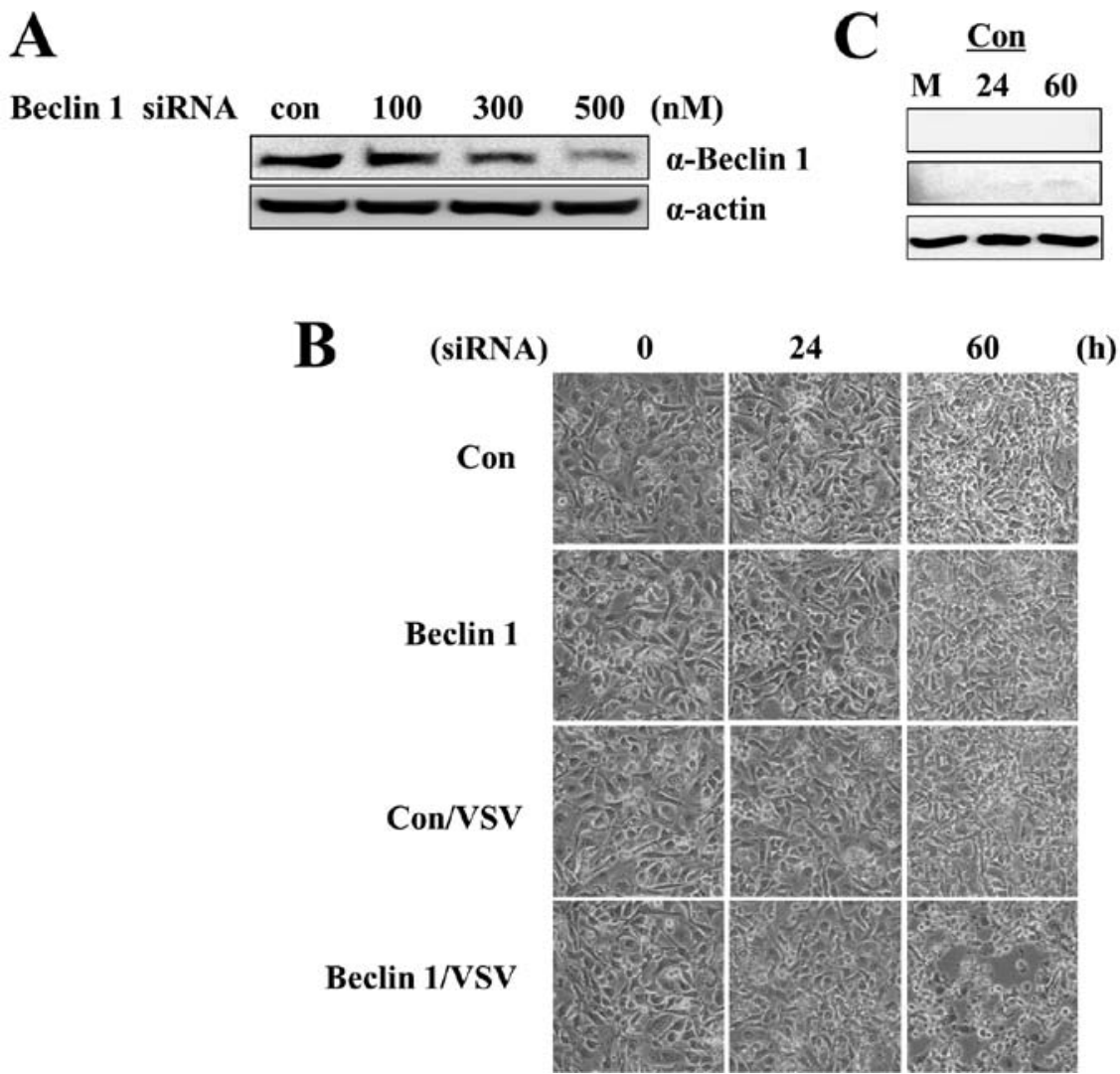

Figure 2. Suppression of Beclin 1 sensitizes A549-CUG2 cells to VSV-induced apoptosis. (A) A549-CUG2 cells were treated with control or Beclin 1 siRNA at different concentrations for $48 \mathrm{~h}$, and the expression of Beclin 1 was examined by immunoblotting with Beclin 1 antibody. (B) A549-CUG2 cells were treated with control or Beclin 1 siRNA at $24 \mathrm{~h}$ before VSV infection (MOI 0.1) and observed at 0,24 , and $60 \mathrm{~h}$ after infection under a light microscope. (C) Cell lysates were prepared and separated on a 10\% SDS-PAGE gel. The expression of PARP and VSV G protein wa observed by immunoblotting with the corresponding antibodies.

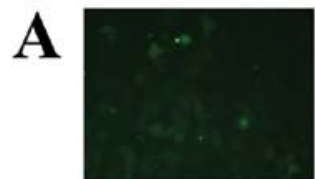

Con

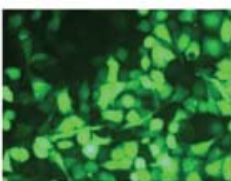

Atg5
Beclin 1 (siRNA/VSV)

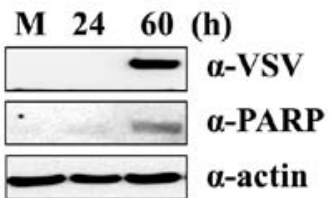

h) 

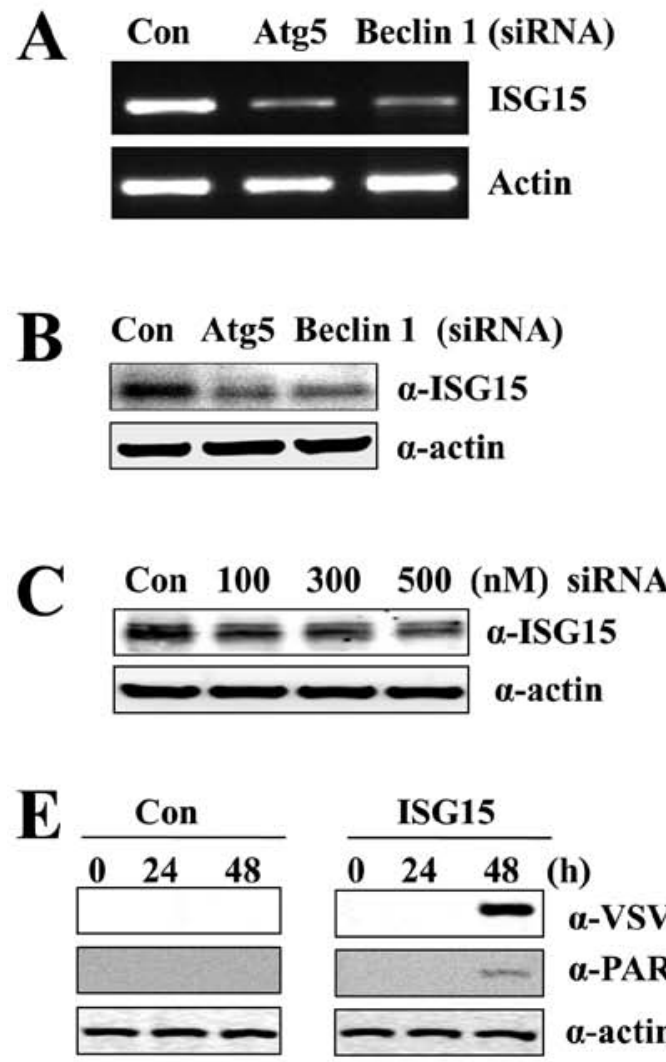

-VSV

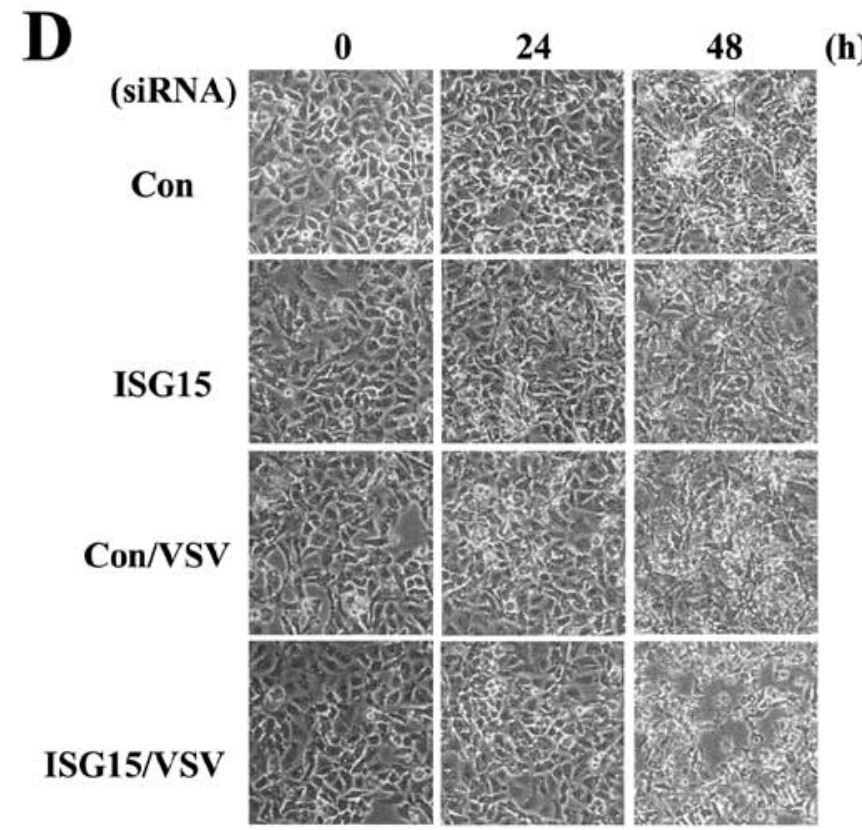

Figure 4. Suppression of ISG15 sensitizes A549-CUG2 cells to VSV-induced apoptosis. (A) Total RNAs isolated from A549-CUG2 cells were treated with control (lane 1), Atg5 (lane 2), or Beclin 1 (lane 3) siRNA at $500 \mathrm{nM}$ for $48 \mathrm{~h}$ and then were subjected to cDNA synthesis with reverse transcriptase. PCR was run at the optimized cycle number using each primer. (B) A549-CUG2 cells were treated with control, Atg5, or Beclin 1 siRNA at 500 nM for 48 h, and the expression of ISG15 was examined by immunoblotting with ISG15 antibody. (C) A549-CUG2 cells were treated with control or ISG15 siRNA at different concentrations for $48 \mathrm{~h}$, and the expression of ISG15 was examined by immunoblotting with ISG15 antibody. (D) A549-CUG2 cells were treated with control or ISG15 siRNA at $24 \mathrm{~h}$ before VSV infection (MOI 0.1) and observed at 0,24 , and $48 \mathrm{~h}$ after infection under a light microscope. (E) Cell lysates were prepared and separated on a 10\% SDS-PAGE gel. The expression of PARP and VSV G protein was observed by immunoblotting with the corresponding antibodies.

ROS formation due to suppression of autophagic gene expression and reduction of antiviral activity. Our previous study revealed an upregulation of antiviral genes such as OASL2 and ISG15. OASL2 expression showed more dependence on Stat1 activation than did ISG15 expression (5). We thus examined the transcript levels of ISG15 after treatment with Atg5 or Beclin 1 siRNA. We found that the ISG15 transcript levels were reduced after treatment with Atg5 or Beclin 1 siRNA compared to those in the cells treated with control siRNA (Fig. 4A and B).The decreased ISG15 transcript level led to suppression of ISG15 protein expression under Atg5- or Beclin 1-deficient conditions. Therefore, we investigated whether the reduced expression of ISG15 transcripts sensitized A549-CUG2 cells to VSV infection. We determined the optimum concentration of ISG15 siRNA to be $500 \mathrm{nM}$ for the suppression of ISG15 transcript expression (Fig. 4C). Treatment with VSV and ISG15 siRNA sensitized A549-CUG2 cells to VSV-induced apoptosis (Fig. 4D and E) caused by VSV replication; on the other hand, VSV infection failed to induce apoptosis in control siRNA-treated A549-CUG2 cells, as shown in Figs. 1B and 2B. This result indicates that suppression of ISG15 by Atg 5 or Beclin 1 siRNA renders A549-CUG2 cells susceptible to VSV infection. Taken together, these results suggest that suppression of autophagy leads to excessive ROS, which impairs the cell's innate immunity, thus rendering it susceptible to VSV infection.

ROS sensitizes A549-CUG2 cells to VSV-induced apoptosis. On the basis of our previous results (Figs. 1, 2 and 4), we hypothesized that ROS formation, caused by the accumulation of damaged mitochondria due to deficiency in autophagic genes, reduces ISG15 protein levels in A549-CUG2 cells, leading to VSV-induced apoptosis. To test this hypothesis, we treated A549-CUG2 cells with different concentrations of $\mathrm{H}_{2} \mathrm{O}_{2} . \mathrm{H}_{2} \mathrm{O}_{2}$ at $50 \mu \mathrm{M}$ significantly decreased ISG15 protein levels (Fig. 5A). A549-CUG2 cells treated with VSV and $\mathrm{H}_{2} \mathrm{O}_{2}$ were susceptible to VSV-induced apoptosis (Fig. 5B and $\mathrm{C}$ ) caused by VSV replication. However, VSV infection or $\mathrm{H}_{2} \mathrm{O}_{2}$ treatment alone did not induce apoptosis. This result confirms that ROS formation sensitizes A549-CUG2 cells to VSV-induced apoptosis.

\section{Discussion}

Autophagy has been considered as an intrinsic antiviral mechanism against viral infection (13-15). The autophagic process allows sequestration of the virus inside the autophagosome, leading to their destruction in the lysosome (24). Autophagy is also involved in the digestion of endogenously synthesized 

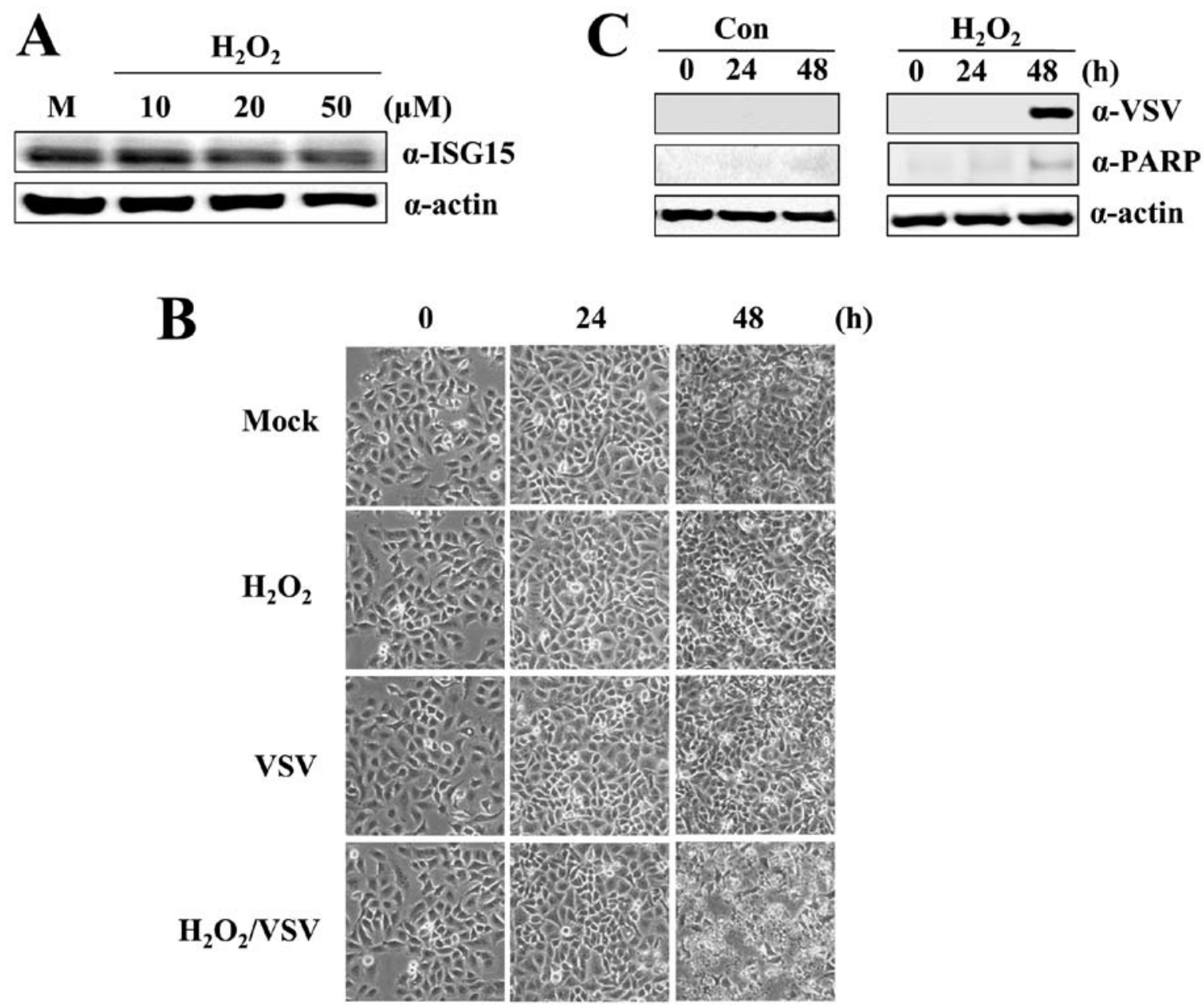

Figure 5. $\mathrm{H}_{2} \mathrm{O}_{2}$ overloading sensitizes A549-CUG2 cells to VSV-induced apoptosis through downregulation of ISG15. (A) A549-CUG2 cells were treated with $\mathrm{H}_{2} \mathrm{O}_{2}$ at the different concentrations for $48 \mathrm{~h}$ and the expression of ISG15 was examined by immunoblotting with ISG15 antibody. (B) A549-CUG2 cells were treated with $50 \mu \mathrm{M} \mathrm{H}_{2} \mathrm{O}_{2}$, infected with VSV (MOI 0.1), and observed at 0, 24, and $48 \mathrm{~h} \mathrm{after}$ infection under a light microscope. (C) Cell lysates were prepared and separated on a 10\% SDS-PAGE gel. The expression of PARP and VSV G protein were observed by immunoblotting with the corresponding antibodies.

viral proteins for MHC II presentation of viral antigens, reflecting the role of autophagy in adaptive immunity (25). Recent evidence also shows that autophagy is linked to type I IFN induction through TLR-7 in the cell's antiviral response (17). However, viruses also have evolved strategies to escape antiviral autophagy. For example, the $\alpha$-herpesvirus HSV-1 ICP34.5 inhibits the induction of autophagy by targeting Beclin 1 and PKR-mediated phosphorylation of eIF $2 \alpha(26,27)$. KSHV and $\gamma$-HV68 viral Bcl-2 proteins attenuate autophagy through direct interaction with Beclin 1 $(28,29)$. Additionally, influenza A virus matrix protein 2 (M2), a proton-selective ion channel, has been identified to be necessary and sufficient for the inhibition of autophagosomal maturation (30). Mouse hepatitis virus (MHV) uses autophagosome-like structures for its production (31).

In the case of VSV, autophagy stimulates IFN $\alpha$ secretion by $\mathrm{pDCs}$ in response to VSV recognition by TLR-7 in mice (17). In vivo, autophagy protects against lethal infection in Drosophila and inhibits viral replication in Drosophila cells (32). However, autophagy negatively regulates RIG-Imediated induction of type I IFN in VSV-infected MEFs.
In particular, autophagy-defective $\operatorname{Atg} 5^{-/-} \mathrm{MEF}$ cells exhibit enhanced RLR signaling, increased IFN secretion and resistance to VSV infection $(20,21)$. In contrast, we observed VSV susceptibility in A549-CUG2 cells with suppressed Atg5 expression. ROS formation is a common phenomenon observed in Atg5 ${ }^{-/-}$MEF cells and A549-CUG2 cells treated with Atg5 siRNA; however, differential results were observed for VSV infection. We assume that this discrepancy might be attributed to cellular context, but we are investigating in more detail the mechanism(s) whereby autophagy impairment induces sensitization of A549-CUG2 cells to VSV.

Autophagy impairment could induce premature senescence in human primary fibroblasts through p53 activation due to increased ROS formation resulting from accumulation of dysfunctional mitochondria. Further investigation revealed that phosphorylation of S6 kinase and ribosomal S6 protein was decreased due to ROS-caused autophagy impairment (33). Consistent with these results, we observed a decrease in phospho-S6 kinase levels due to autophagy impairment. Moreover, one study reported that mTOR signaling stimulates type I IFN production via phosphorylation of its target 
proteins 4E-BPs and S6 kinase 1/2 (34). MEF cells and mice lacking S6 kinase were more susceptible to VSV infection than their WT counterparts as a result of an impaired type I IFN response (23). On the basis of these results, we speculate that decreased levels of phospho-S6 kinase leads to reduced IFN production, which eventually results in the sensitization to VSV of A549-CUG2 cells treated with Atg5 or Beclin 1 siRNA.

In addition, we observed that ROS caused by autophagy impairment led to downregulatiion of ISG15 expression levels in A549-CUG2 cells. The antiviral role of ISG15 has been supported by the observations that the overexpression of ISG15 represses Sindbis virus replication in multiple organs of IFN receptor-deficient mice and protects the host against virus-induced lethality (35). Several IFN-induced antiviral proteins were recently identified as cellular targets of ISG15, including PKR, MxA, and RIG-I, suggesting that ISG15 conjugation may play an important regulatory role in the IFN-mediated antiviral response $(36,37)$. However, ISG15deficient mice were initially reported to exhibit normal IFN signaling and resistance to VSV infection (38). In this study, we showed that suppression of ISG15 by siRNA sensitized A549-CUG2 cells to VSV infection. This discrepancy in the ISG15 antiviral ability may be attributed to the experimental setting. We found only a few antiviral genes such as OASL2, ISG15, IFIT3, IP-10 and GBP3 to be upregulated in the microarray analysis. Among them, OASL2 (strongly dependent on Stat1 activation) and ISG15 (less dependent on Stat1 activation) were the most abundant (5) so that suppression of ISG15, a major component of antiviral protein in A549-CUG2 cells, may reduce the threshold against viral infectivity. We thus propose that CUG2-mediated antiviral activity can be reduced by ISG15 deficiency in A549 cells, which eventually enhances sensitivity of the cells to VSV infection. This study revealed that autophagy with intrinsic antiviral activity affects the efficiency of VSV treatment on tumors overexpressing CUG2. Not only activation of Stat1 but also autophagy as an antiviral mechanism can cause difficulties in virotherapy. Therefore, we suggest that autophagy impairment can be a potential strategy for VSV treatment of tumor cells overexpressing CUG2.

\section{Acknowledgements}

This study was supported by a grant from the National R\&D Program for Cancer Control, Ministry of Health \& Welfare, Republic of Korea (1120140).

\section{References}

1. Lee S, Gang J, Jeon SB, et al: Molecular cloning and functional analysis of a novel oncogene, cancer-upregulated gene 2 (CUG2). Biochem Biophys Res Commun 360: 633-639, 2007.

2. Kim H, Lee M, Lee S, et al: Cancer-upregulated gene 2 (CUG2), a new component of centromere complex, is required for kinetochore function. Mol Cells 27: 697-701, 2009.

3. Park EH, Park EH, Cho IR, et al: CUG2, a novel oncogene confers reoviral replication through Ras and p38 signaling pathway. Cancer Gene Ther 17: 307-314, 2010.

4. Malilas W, Koh SS, Kim S, et al: Cancer upregulated gene 2, a novel oncogene, enhances migration and drug resistance of colon cancer cells via STAT1 activation. Int J Oncol 43: 11111116,2013
5. Malilas W, Koh SS, Srisuttee R, et al: Cancer upregulated gene 2 , a novel oncogene, confers resistance to oncolytic vesicular stomatitis virus through STAT1-OASL2 signaling. Cancer Gene Ther 20: 125-132, 2013

6. Klionsky DJ and Emr SD: Autophagy as a regulated pathway of cellular degradation. Science 290: 1717-1721, 2000.

7. Levine B and Klionsky DJ: Development by self-digestion: molecular mechanisms and biological functions of autophagy. Dev Cell 6: 463-477, 2004.

8. Levine B and Kroemer G: Autophagy in the pathogenesis of disease. Cell 132: 27-42, 2008

9. Shintani T and Klionsky DJ: Autophagy in health and disease: a double-edged sword. Science 306: 990-995, 2004.

10. Qu X, Yu J, Bhagat G, et al: Promotion of tumorigenesis by heterozygous disruption of the Beclin 1 autophagy gene. J Clin Invest 112: 1809-1820, 2003.

11. Levine B: Cell biology: autophagy and cancer. Nature 446: 745-747, 2007.

12. Takamura A, Komatsu M, Hara T, et al: Autophagy-deficient mice develop multiple liver tumors. Genes Dev 25: 795-800, 2011.

13. Espert L, Codogno P and Biard-Piechaczyk M: Involvement of autophagy in viral infections: antiviral function and subversion by viruses. J Mol Med 85: 811-823, 2007.

14. Shoji-Kawata $S$ and Levine B: Autophagy, antiviral immunity, and viral countermeasures. Biochim Biophys Acta 1793: 1478-1484, 2009.

15. Levine B and Deretic V: Unveiling the roles of autophagy in innate and adaptive immunity. Nat Rev Immunol 7: 767-777, 2007.

16. Mulvey M, Poppers J, Sternberg D and Mohr I: Regulation of eIF2alpha phosphorylation by different functions that act during discrete phases in the herpes simplex virus type 1 life cycle. J Virol 77: 10917-10928, 2003.

17. Lee HK, Lund JM, Ramanathan B, Mizushima N and Iwasaki A: Autophagy-dependent viral recognition by plasmacytoid dendritic cells. Science 315: 1398-1401, 2007.

18. Yoneyama M, Kikuchi M, Natsukawa T, et al: The RNA helicase RIG-I has an essential function in double-stranded RNA-induced innate antiviral responses. Nat Immunol 5: 730-737, 2004.

19. Kato H, Takeuchi O, Sato S, et al: Differential roles of MDA5 and RIG-I helicases in the recognition of RNA viruses. Nature 441: 101-105, 2006.

20. Jounai N, Takeshita F, Kobiyama K, et al: The Atg5 Atg12 conjugate associates with innate antiviral immune responses. Proc Natl Acad Sci USA 104: 14050-14055, 2007.

21. Tal MC, Sasai M, Lee HK, Yordy B, Shadel GS and Iwasaki A: Absence of autophagy results in reactive oxygen species-dependent amplification of RLR signaling. Proc Natl Acad Sci USA 106: 2770-2775, 2009.

22. Kongara $S$ and Karantza V: The interplay between autophagy and ROS in tumorigenesis. Front Oncol 2: 171, 2012.

23. Alain T, Lun X, Martineau Y, et al: Vesicular stomatitis virus oncolysis is potentiated by impairing mTORC1-dependent type I IFN production. Proc Natl Acad Sci USA 107: 1576-1581, 2010.

24. Schmid D, Dengjel J, Schoor O, Stevanovic S and Munz C: Autophagy in innate and adaptive immunity against intracellular pathogens. J Mol Med 84: 194-202, 2006.

25. Dengjel J, Schoor O, Fischer R, et al: Autophagy promotes MHC class II presentation of peptides from intracellular source proteins. Proc Natl Acad Sci USA 102: 7922-7927, 2005.

26. Talloczy Z, Virgin HW IV and Levine B: PKR-dependent autophagic degradation of herpes simplex virus type 1 . Autophagy 2 : 24-29, 2006.

27. Talloczy Z, Jiang W, Virgin HW IV, et al: Regulation of starvation- and virus-induced autophagy by the eIF2alpha kinase signaling pathway. Proc Natl Acad Sci USA 99: 190-195, 2002.

28. Sinha S, Colbert CL, Becker N, Wei Y and Levine B: Molecular basis of the regulation of Beclin 1-dependent autophagy by the gamma-herpesvirus $68 \mathrm{Bcl}-2$ homolog M11. Autophagy 4: 989-997, 2008.

29. Pattingre S, Tassa A, Qu X, et al: $\mathrm{Bcl}-2$ antiapoptotic proteins inhibit Beclin 1-dependent autophagy. Cell 122: 927-939, 2005.

30. Gannage M, Dormann D, Albrecht R, et al: Matrix protein 2 of influenza A virus blocks autophagosome fusion with lysosomes. Cell Host Microbe 6: 367-380, 2009.

31. Prentice E, Jerome WG, Yoshimori T, Mizushima N and Denison MR: Coronavirus replication complex formation utilizes components of cellular autophagy. J Biol Chem 279: 10136-10141, 2004. 
32. Dreux M and Chisari FV: Viruses and the autophagy machinery. Cell Cycle 9: 1295-1307, 2010.

33. Kang HT, Lee KB, Kim SY, Choi HR and Park SC: Autophagy impairment induces premature senescence in primary human fibroblasts. PLoS One 6: e23367, 2011

34. Cao W, Manicassamy S, Tang H, et al: Toll-like receptormediated induction of type I interferon in plasmacytoid dendritic cells requires the rapamycin-sensitive PI(3)K-mTOR-p70S6K pathway. Nat Immunol 9: 1157-1164, 2008

35. Lenschow DJ, Giannakopoulos NV, Gunn LJ, et al: Identification of interferon-stimulated gene 15 as an antiviral molecule during Sindbis virus infection in vivo. J Virol 79: 13974-13983, 2005
36. Malakhov MP, Kim KI, Malakhova OA, Jacobs BS, Borden EC and Zhang DE: High-throughput immunoblotting. Ubiquitiin-like protein ISG15 modifies key regulators of signal transduction. J Biol Chem 278: 16608-16613, 2003.

37. Zhao C, Denison C, Huibregtse JM, Gygi S and Krug RM: Human ISG15 conjugation targets both IFN-induced and constitutively expressed proteins functioning in diverse cellular pathways. Proc Natl Acad Sci USA 102: 10200-10205, 2005.

38. Osiak A, Utermohlen O, Niendorf S, Horak I and Knobeloch KP: ISG15, an interferon-stimulated ubiquitin-like protein, is not essential for STAT1 signaling and responses against vesicular stomatitis and lymphocytic choriomeningitis virus. Mol Cell Biol 25: 6338-6345, 2005. 